\title{
The total synthesis of D-chalcose and its C-3 epimer
}

\author{
Jun Sun ${ }^{1}$, Song Fan ${ }^{1}$, Zhan Wang ${ }^{1}$, Guoning Zhang ${ }^{1}$, Kai Bao ${ }^{* 2}$ \\ and Weige Zhang ${ }^{* 1}$
}

\author{
Full Research Paper

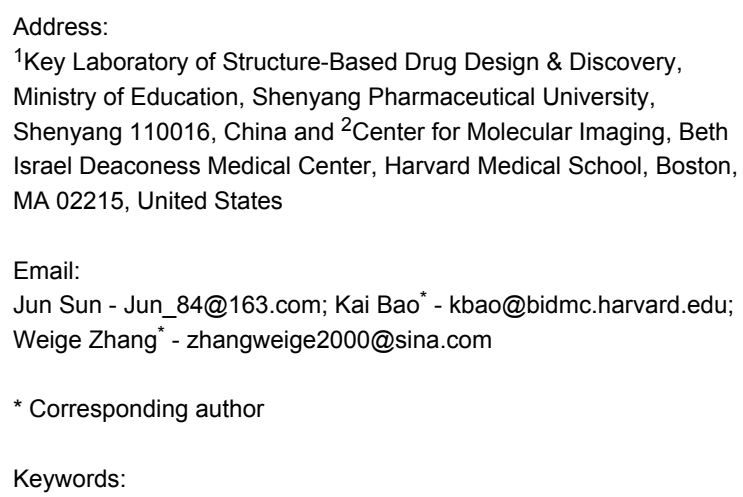 \\ Beilstein J. Org. Chem. 2013, 9, 2620-2624. \\ doi:10.3762/bjoc.9.296 \\ Received: 24 August 2013 \\ Accepted: 07 November 2013 \\ Published: 22 November 2013 \\ Associate Editor: S. Flitsch \\ (c) 2013 Sun et al; licensee Beilstein-Institut. \\ License and terms: see end of document.
}

Open Access

\begin{abstract}
We completed a new and efficient synthesis of D-chalcose (I) and the first synthesis of its C-3 epimer (I') in nine steps with overall yields of $23 \%$ and $24 \%$, respectively. The key steps in the sequence were the formation of the stereocenter on C3 via Grignard reaction, the introduction of the stereogenic center on $\mathrm{C} 2$ by Sharpless asymmetric dihydroxylation, the protection of the $\mathrm{C} 1$ and $\mathrm{C} 2$ hydroxy groups with tert-butyldimethylsilyl trifluoromethanesulfonate (TBSOTf), and the selective cleavage of the primary OTBS ether using catalytic DL-10-camphorsulfonic acid (CSA) in $\mathrm{MeOH}$.
\end{abstract}

\section{Introduction}

Chalcose (4,6-dideoxy-3-O-methyl-D-xylo-hexose, $\mathbf{I}[1,2])$ is a structural component of many macrolide antibiotics, such as chalcomycin [3], neutramycin [4], and lankamycin [5] (Scheme 1). After its structure was determined using chemical degradation and spectroscopic analyses, several syntheses of chalcose were reported. The conversion of desosamine into D-chalcose was described by Westwood's group [6]. A small number of stereospecific syntheses beginning from carbohydrate precursors have been reported [7-10], but the route developed by Usov et al. [11] was especially significant because it demonstrated a facile method to introduce the deoxy functional- ities. Acrolein dimer [12], methyl 2-cis-5-hexadienoate [13], and trans-1-methoxy-3-(trimethylsilyloxy)-1,3-butadiene [14] have been utilized as non-carbohydrate precursors during the synthesis of DL-chalcose, but no attempt was made to resolve the racemate. $\mathrm{D}$ - and L-chalcose were prepared from racemic or meso-divinylglycols by Schmidt et al. $[15,16]$, however, this method had drawbacks, such as non-commercially sourced material and unsatisfactory overall yield. As part of our study concerning the structure-activity relationships and chemistry of new antibiotics, we sought to develop a reliable and efficient synthetic route starting from inexpensive, commercial sources 
to provide access to chalcose. We describe a new and efficient 9 -step synthesis of D-chalcose (I) with a $23 \%$ overall yield. In addition its efficiency, the route enabled facile preparation of chalcose's C-3 epimer (4,6-dideoxy-3-O-methyl-D-ribo-hexose, $\left.I^{\prime}\right)$; the C-3 epimer has not been synthesized previously.

\section{Results and Discussion}

The retrosynthetic analysis of $\mathbf{I}$ and $\mathbf{I}^{\prime}$ is presented in Scheme 1. Diol II and II' arose from a Sharpless asymmetric dihydroxylation that form the $\mathrm{C} 2$ stereogenic center. The installation of the C3 stereocenter on vinyl ether III was proposed to utilize a Grignard reaction followed by chromatographic separation. Aldehyde IV would be generated from commercial ethyl (R)-3hydroxybutyrate (1) via reduction.

The synthesis began by protecting the commercial ethyl $(R)-3$ hydroxybutyrate (1) using a nearly quantitative silylation of the free hydroxy group under classical conditions (TBDPSCl, imidazole, DMF, rt) [17] to form the corresponding ester 2 (Scheme 2). The reduction of 2 using DIBAL-H in anhydrous $\mathrm{CH}_{2} \mathrm{Cl}_{2}$ at $-78{ }^{\circ} \mathrm{C}$ was quenched with methanol at $-30{ }^{\circ} \mathrm{C}$ to provide aldehyde 3 [18]. Subsequently, treating aldehyde 3 with vinylmagnesium bromide in the presence of $\mathrm{CuI}$ at $-78{ }^{\circ} \mathrm{C}$ generated alcohol $\mathbf{4}$ and $\mathbf{4}^{\prime}$ as a 4:5 mixture of diastereomers [19]. This mixture was separated using silica gel chromatography to achieve a $78 \%$ overall yield over three steps. The unwanted syn-diastereomer 4' was converted into the required anti-diastereomer $\mathbf{4}$ via Mitsunobu inversion followed by removal of the benzoate group under basic conditions [20]. Similarly, alcohol 4 could be converted to $4^{\prime}$.

Alcohol 4 was methylated using MeI and $t$-BuOK to produce 5 in nearly quantitative yield (Scheme 3 ). Sharpless asymmetric dihydroxylation [21] of olefin 5 using $A D-m i x-\beta$ in a 1:1 mixture of $t-\mathrm{BuOH} / \mathrm{H}_{2} \mathrm{O}$ at $0{ }^{\circ} \mathrm{C}$ over four days afforded diol 6 with $S$ configuration in $80 \%$ yield.

Next, to selectively oxidize the primary alcohol into an aldehyde, we utilized TEMPO/NaClO [22] to oxidize diol 6. However, the reaction failed to deliver aldehyde 7 (Scheme 3 ). Therefore, we proposed that diol $\mathbf{6}$ should be protected and the primary $\mathrm{OH}$ group could then be selectively deprotected and oxidized to yield the corresponding aldehyde. Our first step toward this goal was to select appropriate protective groups. Diol 6 was initially converted into benzylidene derivative $\mathbf{8}$ ( $\alpha, \alpha$-dimethoxytoluene/PPTS/ $\left.\mathrm{CH}_{2} \mathrm{Cl}_{2}\right) ; \mathbf{8}$ was subjected to a<smiles>C#C[C@H](O)C[C@H](OC)[C@H](O)C=O</smiles><smiles>C#C[C@H](O)C[C@@H](OC)[C@@H](O)C=O</smiles>

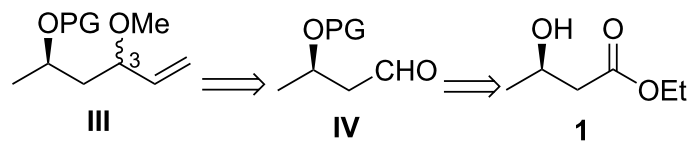

Scheme 1: Retrosynthesis of $I$ and $I ' . P G$ = protecting group; protecting groups may vary independently.

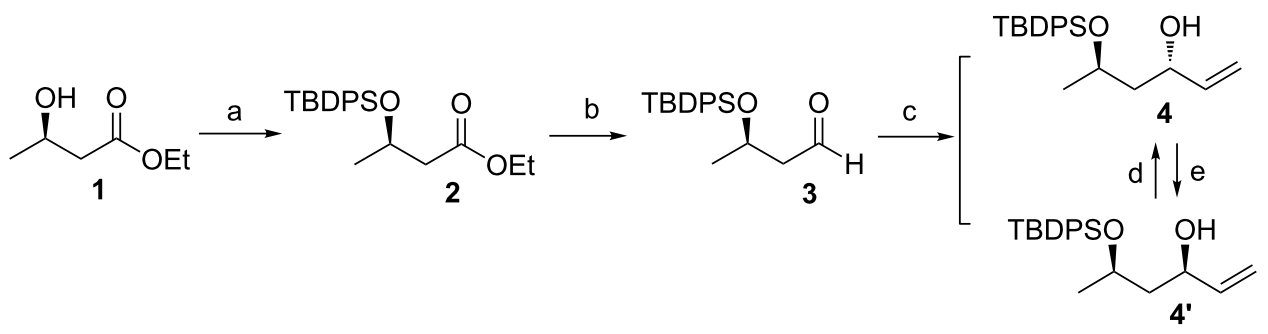

Scheme 2: Reagents and conditions: (a) TBDPSCl, DMAP, imidazole, $\mathrm{CH}_{2} \mathrm{Cl}_{2}$, rt, $99 \%$; (b) DIBAL- $\mathrm{H}, \mathrm{CH}_{2} \mathrm{Cl}_{2},-78{ }^{\circ} \mathrm{C}, 93 \%$; (c) vinylmagnesium bromide, Cul, Et $\mathrm{E}_{2} \mathrm{O},-78{ }^{\circ} \mathrm{C}, 85 \%$; (d) 1) DIAD, $\mathrm{PPh}_{3}, \mathrm{PhCOOH}, \mathrm{THF}, 40{ }^{\circ} \mathrm{C} ; 2$ ) $10 \% \mathrm{NaOH}$, THF, rt, $90 \%$ in two steps; (e) DEAD, $\mathrm{PPh}_{3}, \mathrm{PhCOOH}^{\mathrm{T}} \mathrm{THF}$, $40{ }^{\circ} \mathrm{C}$; 2) $10 \% \mathrm{NaOH}, \mathrm{THF}, \mathrm{rt}, 91 \%$ in two steps. TBDPS $=t$-butyldiphenylsilyl, DMAP $=N, N$-4-dimethylaminopyridine, DIBAL-H = diisobutylaluminum hydride, DIAD = diisopropyl azodicarboxylate, $\mathrm{DEAD}=$ diethyl azodicarboxylate. 

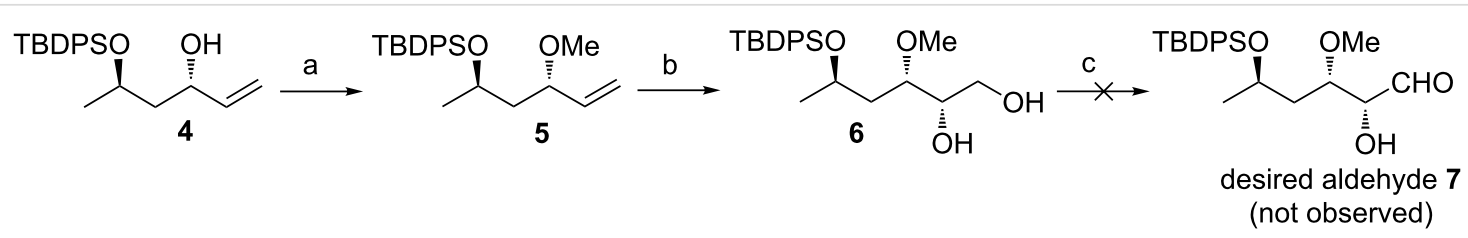

Scheme 3: Reagents and conditions: (a) Mel, $t$-BuOK,THF, rt, $96 \%$; (b) AD-mix- $\beta, t-\mathrm{BuOH} / \mathrm{H}_{2} \mathrm{O}, 0{ }^{\circ} \mathrm{C}, 80 \%$; (c) $\mathrm{TEMPO}, \mathrm{NaClO}, \mathrm{CH} \mathrm{Cl}_{2},-5{ }^{\circ} \mathrm{C}$. $A D=$ asymmetric dihydroxylation, $T E M P O=2,2,6,6$-tetramethyl-1-piperidinyloxy, free radical.

regioselective reductive ring-opening reaction [23] with DIBAL-H in $\mathrm{CH}_{2} \mathrm{Cl}_{2}$ to afford a 1:1 mixture of free primary alcohol $\mathbf{9}$ and undesired secondary alcohol $\mathbf{1 0}$ (Scheme 4).

Consequently, we attempted to treat diol 6 with 2.5 equiv TBSOTf in anhydrous $\mathrm{CH}_{2} \mathrm{Cl}_{2}$ in the presence of 2,6-lutidine for $12 \mathrm{~h}$ at room temperature, providing compound $\mathbf{1 1}$ in $90 \%$ yield [24]. Subsequently, we examined the deprotection of $\mathbf{1 1}$ using the various conditions listed in Table 1. Compound $\mathbf{1 1}$ was initially treated with $\mathrm{PPTS} / \mathrm{MeOH}$ [25] (Table 1 , entry 1 and 2) to form alcohol $\mathbf{1 2}$ as the major expected product. Unfortunately, this reaction was very low yielding and afforded diol $\mathbf{6}$ as the major product. In addition, we used HF-pyridine/THF [26] (Table 1, entry 3 and 4) with similar effect. Treating 11 with $\mathrm{AcOH} / \mathrm{H}_{2} \mathrm{O} / \mathrm{THF}$ (13:7:3) [27] (Table 1, entry 5 and 6) very slowly afforded alcohol 12 in low yield. Eventually, acidcatalyzed deprotection conditions were examined using CSA [28] (Table 1, entry 7-11) in various solvents. After several attempts, we discovered that treating 11 with CSA ( 0.1 equiv) in methanol at $0{ }^{\circ} \mathrm{C}$ for 45 min provided alcohol 12 in the highest yield (Table 1, entry 10). Additionally, the combined yield increased with increasing reaction times, but, the amount of the desired alcohol 12 decreased (Table 1, entries 9 and 10). Moreover, using a protic solvent, such as methanol, was benefi-

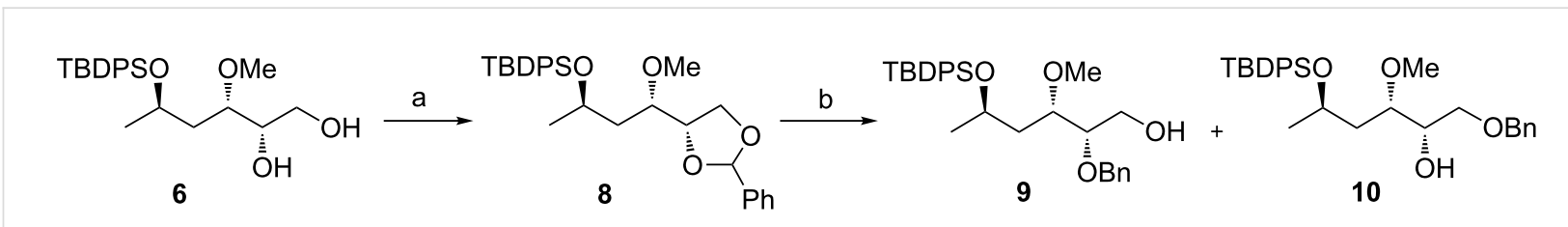

Scheme 4: Reagents and conditions: (a) $\mathrm{PhCH}\left(\mathrm{OCH}_{3}\right)_{2}$, PPTS, $\mathrm{CH}_{2} \mathrm{Cl}_{2}$, rt; (b) DIBAL-H, $\mathrm{CH}_{2} \mathrm{Cl}_{2}, 0{ }^{\circ} \mathrm{C}, 76 \%$ in two steps. PPTS = pyridinium p-toluenesulfonate.

Table 1: Deprotection of 11.

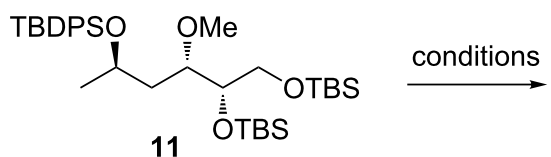

11

\begin{tabular}{|c|c|c|c|}
\hline Entry & Conditions & Yield $(\%)^{a}$ & Ratio $(12: 6)^{b}$ \\
\hline 1 & PPTS (0.1 equiv), $\mathrm{MeOH}, 0^{\circ} \mathrm{C}, 2 \mathrm{~h}$ & trace & \\
\hline 2 & PPTS ( 0.1 equiv), $\mathrm{MeOH}, 25^{\circ} \mathrm{C}, 5 \mathrm{~h}$ & 11 & $0.5: 1$ \\
\hline 3 & HF-pyridine, $\mathrm{THF}, 0^{\circ} \mathrm{C}, 1 \mathrm{~h}$ & trace & \\
\hline 4 & HF-pyridine, THF, $25^{\circ} \mathrm{C}, 1 \mathrm{~h}$ & 17 & $0.2: 1$ \\
\hline 5 & $\mathrm{AcOH}: \mathrm{H}_{2} \mathrm{O}: \operatorname{THF}(13: 7: 3), 25^{\circ} \mathrm{C}, 2 \mathrm{~d}$ & 16 & $1.2: 1$ \\
\hline 6 & $\mathrm{AcOH}: \mathrm{H}_{2} \mathrm{O}: \mathrm{THF}(13: 7: 3), 40^{\circ} \mathrm{C}, 20 \mathrm{~h}$ & 23 & $0.4: 1$ \\
\hline 7 & $\mathrm{CSA}$ (0.1 equiv), $\mathrm{CH}_{2} \mathrm{Cl}_{2}: \mathrm{MeOH}(1: 1), 0{ }^{\circ} \mathrm{C}, 1 \mathrm{~h}$ & 20 & $0.8: 1$ \\
\hline 8 & $\mathrm{CSA}\left(0.1\right.$ equiv), $\mathrm{MeOH}, 0{ }^{\circ} \mathrm{C}, 1 \mathrm{~h}$ & 74 & $2.4: 1$ \\
\hline 9 & CSA (0.1 equiv), $\mathrm{MeOH}, 0{ }^{\circ} \mathrm{C}, 1.2 \mathrm{~h}$ & 76 & $1.5: 1$ \\
\hline 10 & CSA ( 0.1 equiv), $\mathrm{MeOH}, 0{ }^{\circ} \mathrm{C}, 45 \mathrm{~min}$ & 71 & $4.7: 1$ \\
\hline 11 & $\mathrm{CSA}\left(0.1\right.$ equiv), $\mathrm{MeOH}, 0^{\circ} \mathrm{C}, 30 \mathrm{~min}$ & 52 & 4.9:1 \\
\hline
\end{tabular}

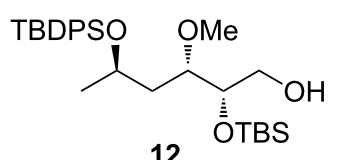

12<smiles>COC(C)CC(OC)[C@@H](O)CO</smiles>

\begin{tabular}{|c|c|c|c|}
\hline Entry & Conditions & Yield $(\%)^{a}$ & Ratio $(12: 6)^{b}$ \\
\hline 1 & PPTS (0.1 equiv), $\mathrm{MeOH}, 0^{\circ} \mathrm{C}, 2 \mathrm{~h}$ & trace & \\
\hline 2 & PPTS ( 0.1 equiv), $\mathrm{MeOH}, 25^{\circ} \mathrm{C}, 5 \mathrm{~h}$ & 11 & $0.5: 1$ \\
\hline 3 & HF-pyridine, $\mathrm{THF}, 0^{\circ} \mathrm{C}, 1 \mathrm{~h}$ & trace & \\
\hline 4 & HF-pyridine, THF, $25^{\circ} \mathrm{C}, 1 \mathrm{~h}$ & 17 & $0.2: 1$ \\
\hline 5 & $\mathrm{AcOH}: \mathrm{H}_{2} \mathrm{O}: \operatorname{THF}(13: 7: 3), 25^{\circ} \mathrm{C}, 2 \mathrm{~d}$ & 16 & $1.2: 1$ \\
\hline 6 & $\mathrm{AcOH}: \mathrm{H}_{2} \mathrm{O}: \mathrm{THF}(13: 7: 3), 40^{\circ} \mathrm{C}, 20 \mathrm{~h}$ & 23 & $0.4: 1$ \\
\hline 7 & $\mathrm{CSA}$ (0.1 equiv), $\mathrm{CH}_{2} \mathrm{Cl}_{2}: \mathrm{MeOH}(1: 1), 0{ }^{\circ} \mathrm{C}, 1 \mathrm{~h}$ & 20 & $0.8: 1$ \\
\hline 8 & $\mathrm{CSA}\left(0.1\right.$ equiv), $\mathrm{MeOH}, 0{ }^{\circ} \mathrm{C}, 1 \mathrm{~h}$ & 74 & $2.4: 1$ \\
\hline 9 & CSA (0.1 equiv), $\mathrm{MeOH}, 0{ }^{\circ} \mathrm{C}, 1.2 \mathrm{~h}$ & 76 & $1.5: 1$ \\
\hline 10 & CSA ( 0.1 equiv), $\mathrm{MeOH}, 0{ }^{\circ} \mathrm{C}, 45 \mathrm{~min}$ & 71 & $4.7: 1$ \\
\hline 11 & $\mathrm{CSA}\left(0.1\right.$ equiv), $\mathrm{MeOH}, 0^{\circ} \mathrm{C}, 30 \mathrm{~min}$ & 52 & 4.9:1 \\
\hline
\end{tabular}

${ }^{\mathrm{a} C}$ Combined yields of $\mathbf{1 2}$ and $\mathbf{6}$. ${ }^{\mathrm{b}}$ The ratios were determined after chromatographic separation. 
cial for the reaction (Table 1, entry 7). Consequently, controlling reaction time was crucial to avoid generating undesired diol 6. Fortunately, diol $\mathbf{6}$ could be recycled via TBSOTf to furnish $\mathbf{1 1}$, and $\mathbf{1 1}$ could be converted into $\mathbf{1 2}$ (Scheme 5).

With requisite intermediate $\mathbf{1 2}$ in hand, our attention was directed toward preparing the final product, chalcose. Alcohol 12 was subjected to Dess-Martin periodinane [29] because it afforded aldehyde $\mathbf{1 3}$ in $86 \%$ yield, making it superior to the Swern oxidation. Subsequently, aldehyde $\mathbf{1 3}$ was efficiently deprotected using TBAF in THF at $0{ }^{\circ} \mathrm{C}$ for $1 \mathrm{~h}$ to form D-chalcose (I) in $84 \%$ yield. Finally, D-chalcose (I) converted into diacetate 14 via treatment with acetic anhydride to facilitate characterization (Scheme 6). The ${ }^{1} \mathrm{H}$ NMR data for diacetate $\mathbf{1 4}$ was identical to the data reported for the natural productderived D-chalcose diacetate [10].

A nearly identical synthetic procedure was used to transform $\mathbf{4}^{\prime}$ into the $\mathrm{C}-3$ epimer of chalcose $\left(\mathbf{I}^{\prime}\right)$; this procedure began with a methylation to form 5' (Scheme 7). The stereoselective dihydroxylation of $\mathbf{5}^{\prime}$ using AD-mix- $\beta$ afforded desired diol $\mathbf{6}^{\prime}$ in
$82 \%$ yield. Subsequently, treating $\mathbf{6}^{\prime}$ with TBSOTf afforded $\mathbf{7}^{\prime}$. Selectively deprotecting the primary hydroxy group using CSA in methanol at $0{ }^{\circ} \mathrm{C}$ for $45 \mathrm{~min}$ provided alcohol $\mathbf{8}^{\prime}$ in $60 \%$ yield, as well as a $15 \%$ yield of diol $\mathbf{6}^{\prime}$ that could be recycled to form 7' using TBSOTf. Alcohol 8' was oxidized using Dess-Martin periodinane to give aldehyde $9^{\prime}$ in $86 \%$ yield. Finally, 9' was efficiently deprotected with TBAF, leading to the C-3 epimer of chalcose $\left(\mathbf{I}^{\prime}\right)$ in $83 \%$ yield. Epimer $\mathbf{I}^{\prime}$ was acetylated using acetic anhydride in pyridine, furnishing diacetate $\mathbf{1 0}^{\prime}$ in $80 \%$ yield; in the major product, $\mathrm{C} 1$ was assigned a $\beta$-configuration based on the NMR spectroscopy data.

\section{Conclusion}

In conclusion, we developed a new and efficient synthesis of D-chalcose (I), requiring nine steps and demonstrating a $23 \%$ overall yield. The first synthesis of its C-3 epimer ( $\left.\mathbf{I}^{\prime}\right)$ was achieved using a similar route in $24 \%$ overall yield. Key epimeric intermediates $\mathbf{4}$ and $\mathbf{4}^{\prime}$ could be interconverted via Mitsunobu reaction, and their absolute configurations were assigned after their transformation into D-chalcose (I) and its C-3 epimer $\left(\mathbf{I}^{\prime}\right)$, respectively. Notably, the stereocenter on C3

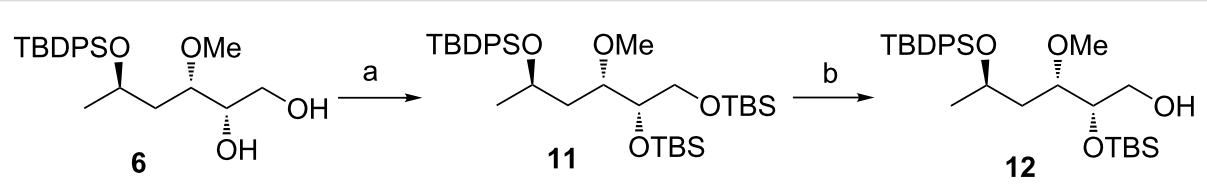

Scheme 5: Reagents and conditions: (a) TBSOTf, 2,6-lutidine, $\mathrm{CH}_{2} \mathrm{Cl}_{2}$, rt, $90 \%$; (b) $\mathrm{CSA}, \mathrm{MeOH}, 0{ }^{\circ} \mathrm{C}, 59 \%$. TBS $=t$-butyldimethylsilyl, $\mathrm{Tf}=$ trifluoromethanesulfonyl, CSA = DL-10-camphorsulfonic acid.

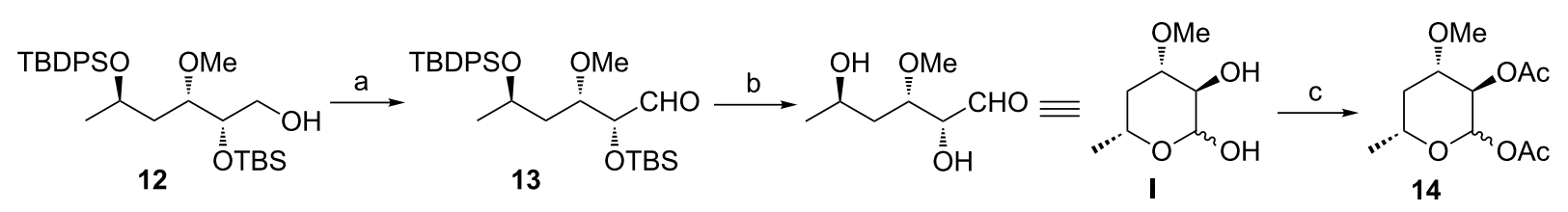

Scheme 6: Reagents and conditions: (a) Dess-Martin periodinane, $\mathrm{CH}_{2} \mathrm{Cl}_{2}$, rt, $86 \%$; (b) TBAF, THF, $0{ }^{\circ} \mathrm{C}, 84 \%$; (c) Py, $\mathrm{AcO} 2$, rt, $80 \%$. TBAF = tetra$n$-butylammonium fluoride.

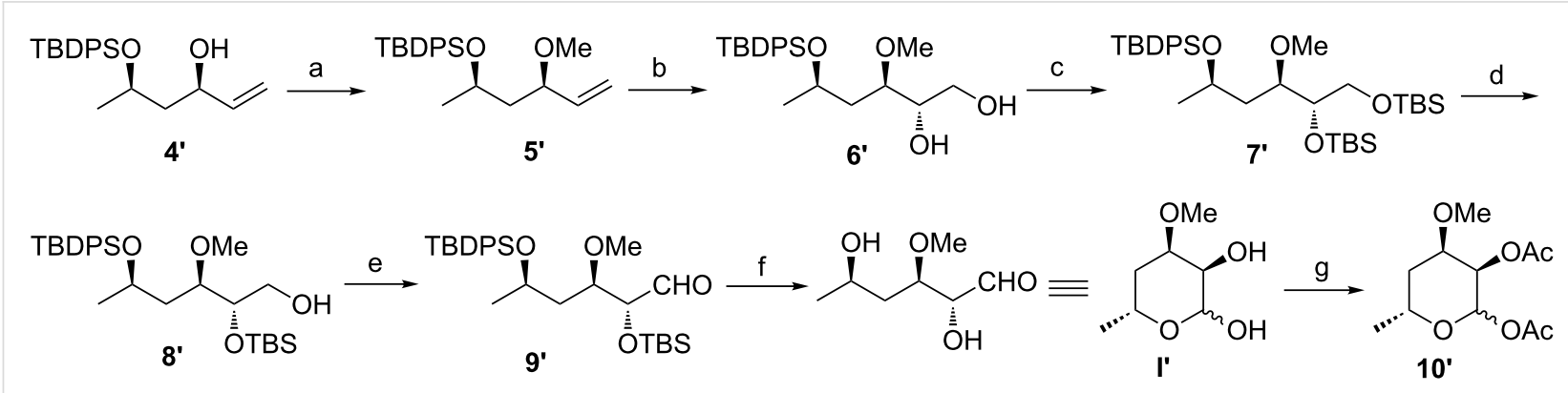


was set using a Grignard reaction, and the hydroxy group on C2 could be introduced with the correct stereochemistry by performing a Sharpless asymmetric dihydroxylation on vinyl ether $\mathbf{5}$. In addition, diol $\mathbf{6}$ was converted to $\mathbf{1 1}$ by protection with TBSOTf followed by selective unveiling of the $\mathrm{C} 1$ hydroxy group via exposure to $\mathrm{CSA} / \mathrm{MeOH}$ for $45 \mathrm{~min}$ at $0{ }^{\circ} \mathrm{C}$; this process afforded alcohol $\mathbf{1 2}$ in good yield. The notable advantage of this strategy is its high degree of flexibility, rendering it applicable to the preparation of various C-3 analogues of chalcose and other related 4-deoxy sugars.

\section{Supporting Information}

\section{Supporting Information File 1}

Experimental details, characterization data of all products, and copies of MS and NMR spectra.

[http://www.beilstein-journals.org/bjoc/content/ supplementary/1860-5397-9-296-S1.pdf]

\section{Acknowledgements}

We gratefully acknowledge the National S\&T Major Project (2012ZX09103101-060), the National Natural Science Foundation of China (Grant No. 30973614), and the Shenyang Science and Technology Bureau Item (No. F12-277-1-23) for their generous financial support.

\section{References}

1. Woo, P. W. K.; Dion, H. W.; Bartz, Q. R. J. Am. Chem. Soc. 1961, 83, 3352-3353. doi:10.1021/ja01476a053

2. Woo, P. W. K.; Dion, H. W.; Johnson, L. F. J. Am. Chem. Soc. 1962, 84, 1066-1067. doi:10.1021/ja00865a049

3. Woo, P. W. K.; Dion, H. W.; Bartz, Q. R. J. Am. Chem. Soc. 1964, 86, 2726-2727. doi:10.1021/ja01067a047

4. Kunstmann, M. P.; Mitscher, L. A. Experientia 1965, 21, 372-373. doi:10.1007/BF02139741

5. Keller-Schierlein, W.; Roncari, G. Helv. Chim. Acta 1962, 45, 138-152. doi:10.1002/hlca.19620450118

6. Foster, A. B.; Stacey, M.; Webber, J. M.; Westwood, J. H. J. Chem. Soc. 1965, 2318-2323. doi:10.1039/JR9650002318

7. McNally, S.; Overend, W. G. Chem. Ind. (London) 1964, 49, 2021.

8. Lawton, B. T.; Ward, D. J.; Szarek, W. A.; Jones, J. K. N. Can. J. Chem. 1969, 47, 2899-2901. doi:10.1139/v69-484

9. Kefurt, K.; Kefurtová, Z.; Jarý, J. Collect. Czech. Chem. Commun. 1973, 38, 2627-2632. doi:10.1135/cccc19732627

10. Redlich, H.; Roy, W. Carbohydr. Chem. 1979, 68, 275-285.

11. Kochetkov, N. K.; Usov, A. I. Tetrahedron Lett. 1963, 4, 519-521. doi:10.1016/S0040-4039(01)90664-1

12. Srivastava, R. M.; Brown, R. K. Can. J. Chem. 1970, 48, 830-837. doi:10.1139/v70-133

13. Torssell, K.; Tyagi, M. P. Acta Chem. Scand., Ser. B 1977, B31, 7-10. doi:10.3891/acta.chem.scand.31b-0007

14. Danishefsky, S.; Kerwin, J. F., Jr. J. Org. Chem. 1982, 47, 1597-1598. doi:10.1021/jo00347a053
15. Küfner, U.; Schmidt, R. R. Angew. Chem. 1986, 98, 90-91. doi:10.1002/ange.19860980116

Angew. Chem., Int. Ed. Engl. 1986, 25, 89

doi:10.1002/anie.198600891

16. Küfner, U.; Schmidt, R. R. Carbohydr. Res. 1987, 161, 211-223. doi:10.1016/S0008-6215(00)90078-8

17. Hanessian, S.; Lavallee, P. Can. J. Chem. 1975, 53, 2975-2977. doi:10.1139/v75-419

18. Massad, S. K.; Hawkins, L. D.; Baker, D. C. J. Org. Chem. 1983, 48, 5180-5182. doi:10.1021/jo00174a006

19. Elsworth, J. D.; Willis, C. L. Chem. Commun. 2008, 1587-1589. doi:10.1039/b717078e

20. Jana, N.; Mahapatra, T.; Nanda, S. Tetrahedron: Asymmetry 2009, 20, 2622-2628. doi:10.1016/j.tetasy.2009.10.007

21. Sharpless, K. B.; Amberg, W.; Bennani, Y. L.; Crispini, G. A.; Hartung, J.; Jeong, K. S.; Kwong, H. L.; Morikawa, K.; Wang, Z. M.; Xu, D.; Zhang, X.-L. J. Org. Chem. 1992, 57, 2768-2771. doi:10.1021/jo00036a003

22. de Nooy, A. E. J.; Besemer, A. C.; van Bekkum, H. Synthesis 1996, 10 1153-1176. doi:10.1055/s-1996-4369

23. Takano, S.; Akiyama, M.; Sato, S.; Ogasawara, K. Chem. Lett. 1983, 12, 1593-1596. doi:10.1246/cl.1983.1593

24. Corey, E. J.; Cho, H.; Rücker, C.; Hua, D. H. Tetrahedron Lett. 1981, 22, 3455-3458. doi:10.1016/S0040-4039(01)81930-4

25. Prakash, C.; Saleh, S.; Blair, I. A. Tetrahedron Lett. 1989, 30, 19-22. doi:10.1016/S0040-4039(01)80311-7

26. Nicolaou, K. C.; Webber, S. E. Synthesis 1986, 453-461. doi:10.1055/s-1986-31673

27. Kawai, A.; Hara, O.; Hamada, Y.; Shioiri, T. Tetrahedron Lett. 1988, 29 , 6331-6334. doi:10.1016/S0040-4039(00)82339-4

28. Hara, A.; Morimoto, R.; Iwasaki, Y.; Saitoh, T.; Ishikawa, Y.; Nishiyama, S. Angew. Chem., Int. Ed. 2012, 51, 9877-9880. doi:10.1002/anie.201204992

29. Dess, D. B.; Martin, J. C. J. Org. Chem. 1983, 48, 4155-4156. doi:10.1021/jo00170a070

\section{License and Terms}

This is an Open Access article under the terms of the Creative Commons Attribution License (http://creativecommons.org/licenses/by/2.0), which permits unrestricted use, distribution, and reproduction in any medium, provided the original work is properly cited.

The license is subject to the Beilstein Journal of Organic Chemistry terms and conditions: (http://www.beilstein-journals.org/bjoc)

The definitive version of this article is the electronic one which can be found at: $\underline{\text { doi: } 10.3762 / \text { bjoc. } 9.296}$ 